\title{
Query refinement for ontology information extraction
}

\begin{abstract}
Ontology information extraction has gain popularity due to the increasing amount of ontologies developed over the years. World Wide Web Consortium (W3C) has introduced SPARQL query language to extract information. However SPARQL query language follow a specific pattern in order to find the triple through subgraph matching. The keywords used in the SPARQL query have to be exactly same as the existing keywords in the RDF data inorder to extract the required information. The paper introduced a method to ease the job of the user by generating SPARQL query from the query entered by the user. The method uses the object property list generated from the ontology and word's synonym to aid in SPARQL query generation. The result has shown that the proposed method clearly eases the need of the user to learn SPARQL syntax.
\end{abstract}

Keyword: Information extraction; Ontology; RDF; SPARQL; Triple 